APJL, IN PRESS

Preprint typeset using $\mathrm{LATE}_{\mathrm{E} X}$ style emulateapj v. 5/25/10

\title{
DISCOVERY OF SMALL-SCALE SPIRAL STRUCTURES IN THE DISK OF SAO 206462 (HD 135344B)* IMPLICATIONS FOR THE PHYSICAL STATE OF THE DISK FROM SPIRAL DENSITY WAVE THEORY
}

T. Muto ${ }^{1,35,36}$, C. A. Grady ${ }^{2,3,4}$, J. Hashimoto ${ }^{5}$, M. Fukagawa ${ }^{6}$, J. B. Hornbeck ${ }^{7}$, M. Sitko ${ }^{8,9,10}$, R. Russell $^{10,11}$, C. Werren ${ }^{9,10}$, M. Curé ${ }^{12}$, T. Currie ${ }^{3}$, N. Ohashi ${ }^{13,14}$, Y. OkAmoto ${ }^{15}$, M. Momose ${ }^{15}$, M. Honda ${ }^{16}$, S. Inutsuka ${ }^{17}$, T.

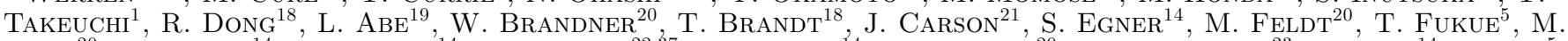

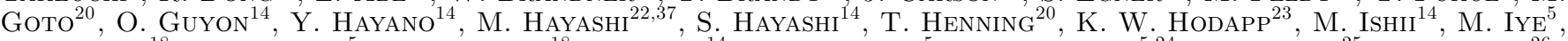
M. Janson ${ }^{18}$, R. Kandori ${ }^{5}$, G. R. Knapp ${ }^{18}$, T. Kudo ${ }^{14}$, N. Kusakabe ${ }^{5}$, M. Kuzuhara ${ }^{5,24}$, T. Matsuo ${ }^{25}$, S. Mayama ${ }^{26}$, M. W. McElwain ${ }^{3}$, S. Miyama ${ }^{5}$, J.-I. Morino ${ }^{5}$, A. Moro-Martin ${ }^{18,27}$, T. Nishimura ${ }^{14}$, T.-S. Pyo ${ }^{14}$, E. Serabyn ${ }^{28}$, H. Suto $^{5}$, R. Suzuki ${ }^{29}$, M. Takami ${ }^{13}$, N. Takato ${ }^{14}$, H. Terada ${ }^{14}$, C. Thalmann ${ }^{30}$, D. Tomono ${ }^{14}$, E. L. Turner ${ }^{18,31}$, M. WAtanabe $^{32}$, J. P. WisnieWski ${ }^{33}$, T. Yamada ${ }^{34}$, H. TAKAmi ${ }^{14}$, T. Usudi ${ }^{14}$, And M. TAmura ApJL, in press

\section{ABSTRACT}

We present high-resolution, $H$-band, imaging observations, collected with Subaru/HiCIAO, of the scattered light from the transitional disk around SAO 206462 (HD 135344B). Although previous sub$\mathrm{mm}$ imagery suggested the existence of the dust-depleted cavity at $r \leq 46 \mathrm{AU}$, our observations reveal the presence of scattered light components as close as $0.22(\sim 28 \mathrm{AU})$ from the star. Moreover, we have discovered two small-scale spiral structures lying within $00^{\prime \prime} 5(\sim 70 \mathrm{AU})$. We present models for the spiral structures using the spiral density wave theory, and derive a disk aspect ratio of $h \sim 0.1$, which is consistent with previous sub-mm observations. This model can potentially give estimates of the temperature and rotation profiles of the disk based on dynamical processes, independently from sub-mm observations. It also predicts the evolution of the spiral structures, which can be observable on timescales of 10-20 years, providing conclusive tests of the model. While we cannot uniquely identify the origin of these spirals, planets embedded in the disk may be capable of exciting the observed morphology. Assuming that this is the case, we can make predictions on the locations and, possibly, the masses of the unseen planets. Such planets may be detected by future multi-wavelengths observations.

Subject headings: circumstellar matter — instrumentation: high angular resolution — polarization — protoplanetary disks — stars: individual (SAO 206462, HD 135344B) — waves

muto@geo.titech.ac.jp

* Based on data collected at the Subaru Telescope, which is operated by the National Astronomical Observatory of Japan.

1 Tokyo Institute of Technology, 2-12-1 Ookayama, Meguro, Tokyo 152-8551, Japan

2 Eureka Scientific, 2452 Delmer, Suite 100, Oakland CA 96002, USA

3 ExoPlanets and Stellar Astrophysics Laboratory, Code 667, Goddard Space Flight Center, Greenbelt, MD 20771 USA

${ }_{5}^{4}$ Goddard Center for Astrobiology, Code 667, Goddard Space Flight Center, Greenbelt, MD 20771 USA

${ }^{5}$ National Astronomical Observatory of Japan, 2-21-1 Osawa, Mitaka, Tokyo 181-8588, Japan

${ }^{6}$ Department of Earth and Space Science, Graduate School of Science, Osaka University, 1-1, Machikaneyama, Toyonaka, Osaka 5600043, Japan

7 Department of Physics and Astronomy, University of Louisville, Louisville, KY 40292, USA

8 Space Science Institute, 4750 Walnut St., Suite 205, Boulder, CO 80301, USA

9 Department of Physics, University of Cincinnati, Cincinnati, OH 45221-0011, USA

10 Visiting Astronomer, NASA Infrared Telescope Facility, operated by the University of Hawaii under contract to NASA

11 The Aerospace Corporation, Los Angeles, CA 90009, USA

12 Departamento de Física y Astronomía, Universidad de Valparaíso, Avda. Gran Bretaña 1111, Casilla 5030, Valparaíso, Chile

13 Institute of Astronomy and Astrophysics, Academia Sinica, P.O. Box 23-141, Taipei 106, Taiwan

14 Subaru Telescope, 650 North A'ohoku Place, Hilo, HI 96720, USA

15 College of Science, Ibaraki University, 2-1-1 Bunkyo, Mito, Ibaraki 310-8512, Japan

16 Department of Information Science, Kanagawa University, 2946 Tsuchiya, Hiratsuka, Kanagawa 259-1293, Japan

17 Department of Physics, Nagoya University, Furo-cho, Chikusa-ku, Nagoya, Aichi, 464-8602, Japan

18 Department of Astrophysical Sciences, Princeton University, NJ08544, USA

19 Laboratoire Lagrange, UMR7293, Université de Nice-Sophia Antipolis, CNRS, Observatoire de la Côte d'Azur, 06300 Nice, France 20 Max Planck Institute for Astronomy, Heidelberg, Germany

${ }^{21}$ Department of Physics and Astronomy, College of Charleston, 58 Coming St., Charleston, SC 29424, USA

22 Department of Astronomy, The University of Tokyo, Hongo 7-3-1, Bunkyo-ku, Tokyo 113-0033, Japan

23 Department of Earth and Planetary Science, The University of Tokyo, Hongo 7-3-1, Bunkyo-ku, Tokyo 113-0033, Japan

${ }^{24}$ Institute for Astronomy, University of Hawaii, 640 North A'ohoku Place, Hilo, HI 96720, USA

25 Department of Astronomy, Kyoto University, Kitashirakawa-Oiwake-cho, Sakyo-ku, Kyoto, 606-8502, Japan

26 The Graduate University for Advanced Studies(SOKENDAI), Shonan International Village, Hayama-cho, Miura-gun, Kanagawa 240-0193, Japan

${ }^{27}$ Departamento de Astrofísica, CAB (INTA-CSIC), Instituto Nacional de Técnica Aeroespacial, Torrejón de Ardoz, 28850, Madrid, Spain

28 Jet Propulsion Laboratory, California Institute of Technology, Pasadena, CA 91109, USA

29 TMT Observatory Corporation, 1111 South Arroyo Parkway, Pasadena, CA 91105, USA

30 Astronomical Institute "Anton Pannekoek", University of Amsterdam, Science Park 904, 1098 XH Amsterdam, The Netherlands

${ }^{31}$ Kavli Institute for the Physics and Mathematics of the Universe, Todai Institutes for Advanced Study, the University of Tokyo, Kashiwa, Japan 277-8583 (Kavli IPMU, WPI)

32 Department of Cosmosciences, Hokkaido University, Sapporo 060-0810, Japan

33 Department of Astronomy, University of Washington, Box 351580 Seattle, Washington 98195, USA

34 Astronomical Institute, Tohoku University, Aoba, Sendai 980-8578, Japan

35 JSPS Research Fellow

36 As of April 2012: Division of Liberal Arts, Kogakuin University, 1-24-2, Nishi-Shinjuku, Shinjuku-ku, Tokyo, 163-8677, Japan 


\section{INTRODUCTION}

Dynamical processes in protoplanetary disks such as turbulence or disk-planet interaction are important in understanding physical condition and evolution of disks, and planet formation processes. High resolution, direct imaging observations of circumstellar/protoplanetary disks can reveal non-axisymmetric structures, providing insight into such dynamical processes (e.g., Hashimoto et al. 2011).

Recent observations have identified a class of protoplanetary disks harboring tens of AU-scale holes/gaps at their centers: the so-called transitional disks. One well-studied system of that class is the rapidly rotating Herbig $\mathrm{F}$ star, SAO 206462 (HD 135344B, F4Ve, $d=142 \pm 27$ pc, $M=1.7_{-0.1}^{+0.2} M_{\odot}$, Müller et al. 2011). The observations of CO line profiles (Dent et al. 2005; Pontoppidan et al. 2008; Lyo et al.2011) and stellar rotation (Müller et al. 2011) consistently indicate an almost face-on geometry $\left(i \sim 1^{\circ}\right)$. The gap in the disk was predicted from the infrared (IR) spectral energy distribution (SED, Brown et al.|2007), and was subsequently imaged in sub-mm dust continuum at $\sim 0$ '. $5 \times 0$ '. 25 resolution (Brown et al. 2009). Andrews et al. (2011) estimate the gap radius to be $\sim 46$ AU and the surface density within the gap to be $10^{-5.2}$ times smaller than that extrapolated from the outer disk. The gas in Keplerian motion surrounding the gap region is also imaged by CO lines (Lvo et al. 2011). The CO rovibrational line observations (Pontoppidan et al. 2008) and [OI] spectral line observations (van der Plas et al. 2008) indicate the presence of a gas disk in the vicinity (several AU-scale) of the star. SED modeling (Grady et al. 2009) and NIR interferometry (Fedele et al. 2008) indicate the presence of an inner dust belt, which is temporally variable (Sitko et al. 2012) and not coplanar with the outer disk (Benisty 2011, private communication). New imaging with high spatial resolution and sensitivity is required to understand the inner structures of the disk. The outer portions of gaps can now be resolved using 8-10 m ground-based telescopes at near infrared (NIR) wavelengths (e.g., Thalmann et al. 2010, for LkCa 15).

In this Letter, we present $H$-band polarized intensity $(P I)$ observations of the disk of SAO 206462 down to $r \sim$ 0.'2 $(\sim 28 \mathrm{AU})$ scale at $0 . \prime 06(\sim 8.4 \mathrm{AU})$ resolution. Interior to the sub-mm resolved gap, we find spiral structures, indicative of dynamical processes. We use the spiral density wave theory to interpret the structure, and estimate disk's physical parameters.

\section{OBSERVATIONS \& DATA REDUCTION}

\subsection{HiCIAO Observations}

SAO 206462 was observed in the $H$-band $(1.6 \mu \mathrm{m})$ using the high-contrast imaging instrument HiCIAO (Tamura et al. 2006; Hodapp et al. 2008; [Suzuki et al. 2010) on the Subaru Telescope on 2011 May 20 UT as part of Strategic Explorations of Exoplanets and Disks with Subaru (SEEDS, Tamura 2009). The adaptive optics system (AO188; Hayano et al. 2004; Minowa et al. 2010) provided a stable stellar point spread function (PSF, FWHM = 0.'06). We used a combined angular differential imaging (ADI) and polarization differential imaging (PDI) mode with a field of view of $10^{\prime \prime} \times 20^{\prime \prime}$ and a pixel scale of $9.5 \mathrm{mas}^{\mathrm{pixel}}{ }^{-1}$. A $0^{\prime \prime}$. 3 -diameter circular occulting mask was used to suppress the bright stellar halo. The half-wave plates were placed to four angular positions from $0^{\circ}, 45^{\circ}, 22.5^{\circ}$, and $67.5^{\circ}$ in sequence with one $30 \mathrm{sec}$ exposure per wave plate position. The total integration time of the $P I$ image was 780 sec after removing low quality images with large FWHMs by careful inspections of the stellar PSF.

\subsection{PDI Data Reduction}

The raw images were corrected using IRAF 1 for dark current and flat-field following the standard reduction scheme. We applied a distortion correction using globular cluster M5 data taken within a few days, using IRAF packages GEOMAP and GEOTRAN. Stokes $(Q, U)$ parameters and the $P I$ image were obtained in the standard approach (e.g., Hinkley et al. 2009) as follows. By subtracting two images of extraordinary- and ordinary-rays at each wave plate position, we obtained $+Q,-Q,+U$, and $-U$ images, from which $2 Q$ and $2 U$ images were made by another subtraction to eliminate remaining aberration. $P I$ was then given by $P I=\sqrt{Q^{2}+U^{2}}$. Instrumental polarization of HiCIAO at the Nasmyth instrument was corrected by following Joos et al. $(2008)$. From frame-by-frame deviations, the typical error of surface brightness (SB) was estimated to be $\sim 5 \%$ at $r \sim 0.5$ when averaged over $5 \times 5$ pixels $(\sim$ PSF scale). Comparing different data reduction methods (frame selections and instrumental polarization estimates), we expect that the systematic uncertainty of the SB of $P I$ to be $\sim 10 \%$.

\subsection{Contemporaneous Photometry}

Since SAO 206462 shows variability in NIR wavelengths (Sitko et al. 2012), it is important to take photometry simultaneously with disk observations. $H$-band photometry was obtained just before and after the disk imaging without the coronagraphic spot with the adaptive optics, by sixteen 1.5 sec exposures at four spatially dithered positions. An ND10 filter $(9.8 \pm 0.1 \%$ transmission) was used to avoid saturation. Using the MKO filter set, the $H$-band $\left(\lambda_{\text {eff }}=1.615 \mu \mathrm{m}, \mathrm{FWHM}=0.29 \mu \mathrm{m}\right.$, Tokunaga et al. 2002) magnitude was $6.96 \pm 0.07 \mathrm{mag}$.

Broad-band VRIJHK photometry was obtained on 2011 May 23-26, starting within 48 hours of the HiCIAO observation, using the Rapid Eye Mount (REM) Telescope at La Silla, Chile (Covino et al. 2004). The REM H-band filter has $\lambda_{\text {eff }}=1.65 \mu \mathrm{m}, \mathrm{FWHM}=0.35 \mu \mathrm{m}$ : broader and displaced to longer wavelengths than the MKO filter. The observed data were reduced differentially using SAO 206463 (A0V). The IR excess due to the inner disk (Figure1) was

${ }^{1}$ IRAF is distributed by the National Optical Astronomy Observatory, which is operated by the Association of Universities for Research in Astronomy, Inc., under cooperative agreement with the National Science Foundation. 


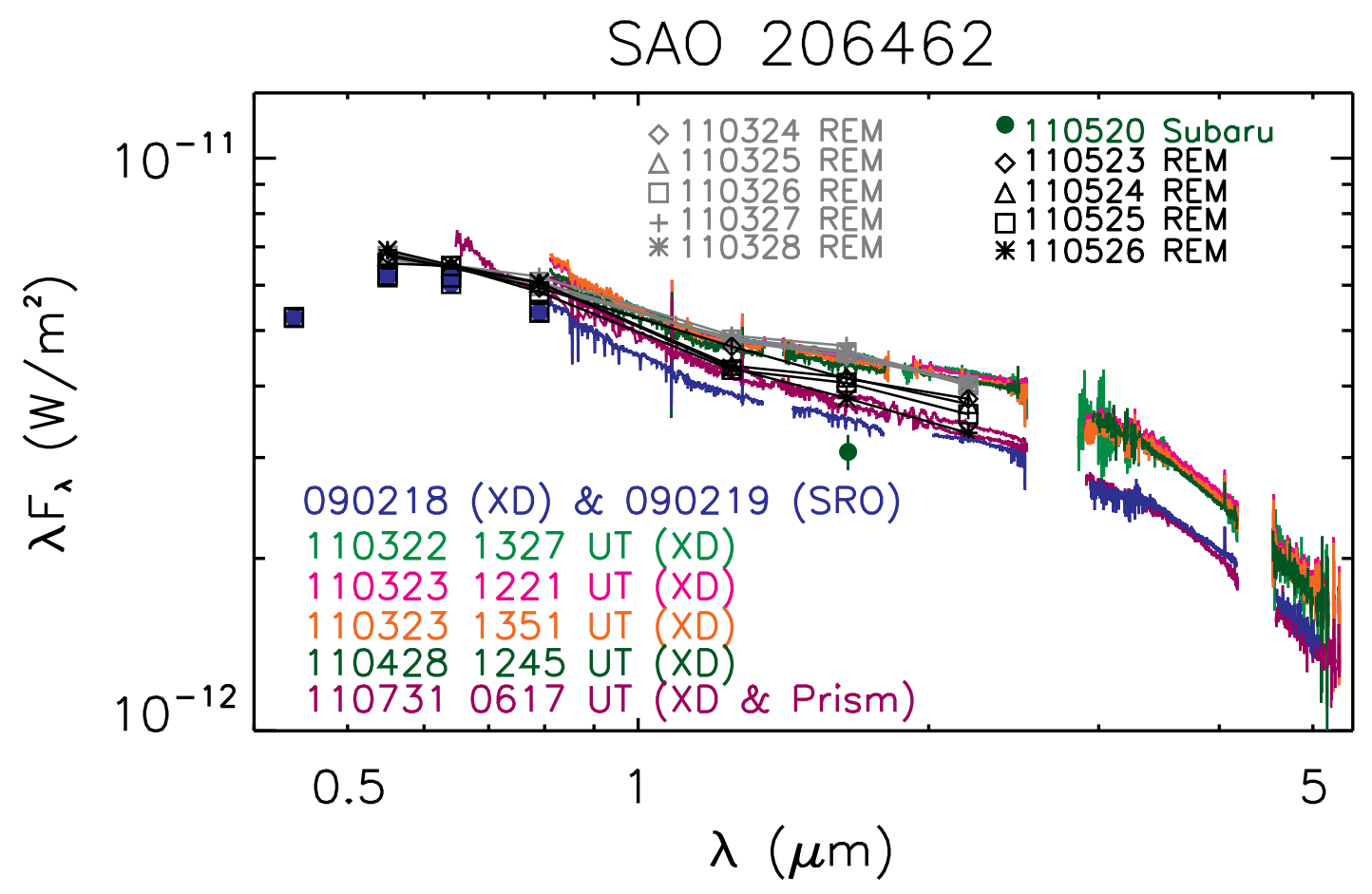

FIG. 1. - SED for SAO 206462 obtained by REM, adapted, in part, from Sitko et al. (2012). The REM observations consist of VRIJHK photometry. Also shown are spectra obtained with the SpeX spectrograph. See Section 2.3 for the data reduction techniques.

average for the range observed in 2009-2011 (Sitko et al.|2012). No significant variation was observed during the 2011 May observations, except for the small long-term fading trend $(\delta \mathrm{m}=0.08 \pm 0.02 \mathrm{mag})$ over the observation period.

Figure 1 also displays spectra obtained with the SpeX spectrograph (Rayner et al.|2009) on NASA's Infrared Telescope Facility (IRTF). The observations were obtained in the cross-dispersed (XD) echelle mode between 0.8 and $5.1 \mu \mathrm{m}$ using a $0^{\prime \prime} 8$ slit $(R \sim 900)$ and calibrated using HD 129685 (A0V) with SpeXtool (Vacca et al. 2003; Cushing et al. 2004). The absolute flux calibration, to correct for light loss at the spectrograph slits, was accomplished in two ways: using photometry and wide-slit spectroscopy (see Sitko et al. 2012). The March SpeX data were normalized using the REM photometry, obtained in the days immediately after the SpeX observations, and when the star was photometrically stable. In July, SAO 206462 and the calibration star were observed with the SpeX prism using a 3"'0 slit, which, under good seeing and transparency conditions, produces absolute flux to $\sim 5 \%$ accuracy. The REM photometry at $H$-band in May is $\sim 0.25$ mag brighter than the Subaru data. The Subaru photometry shows a low value even when considering the filter difference, suggesting that the outer disk is illuminated efficiently.

\section{RESULTS}

\subsection{Spiral Structure}

The SAO 206462 disk can be traced in PI from $0^{\prime \prime} 2$ to $\sim 11^{\prime \prime} 0(28-140 \mathrm{AU})$, similar to the range imaged with HST/NICMOS (Grady et al. 2009), but with a factor of $\sim 4$ greater angular resolution. The total PI is $9.87 \mathrm{mJy} \pm$ $0.06 \%$ at $0^{\prime \prime} .2<r<1^{\prime \prime} .0$, which is $0.6 \%$ of the stellar intensity. The total $P I$ at $0^{\prime \prime} .42<r<1^{\prime \prime} .0$ is $3.94 \mathrm{mJy} \pm 0.1 \%$ while the total intensity by HST/NICMOS F110W is $9.7 \mathrm{mJy}$ (Grady et al. 2009). The average SB of $P I$ at $r=00^{\prime \prime} 46$ is $\sim 6 \mathrm{mJy} / \mathrm{asec}^{2}$, whereas the total intensity by HST/NICMOS F160W is $30 \mathrm{mJy} / \mathrm{asec}^{2}$ (Grady et al. 2009). Given the NICMOS data uncertainties, the polarization fraction is $\sim 20-40 \%$, assuming no PSF halo in the HiCIAO data and no variable self-shadowing/illumination in the disk. Our measured polarization fraction is similar to that of HD $100546\left(14 \%_{-8 \%}^{+19 \%}\right.$, Quanz et al. 2011) and AB Aur ( 25\% - 45\%, Perrin et al. 2009).

Figure 2 shows the PI image. The region interior to $0{ }^{\prime \prime} 4$ is not a void and we do not see clear structural evidence of the cavity wall in Andrews et al. (2011) model $\left(R_{\text {cav }}=46 \mathrm{AU} \sim 0^{\prime \prime} 33\right)$. We see spiral arcs S1 (east) and S2 (southwest). The $P I$ at the location of the spirals is $\sim 30 \%$ larger than that extrapolated from the smooth outer profile (bottom of Figure 21). The brightest portions of the spirals roughly coincide with the bright thermal emission peaks at $12 \mu \mathrm{m}$ (Mariñas et al. 2011) and lie inside the ring noted by Doucet et al. (2006). It is also noted that we see a dip in $P I$ in the north-west, probably due to the depolarization in the minor axis direction (see below), and that we do not see large-scale, localized shadow that might be cast by the inner dust belt if highly inclined relative to the outer disk. 

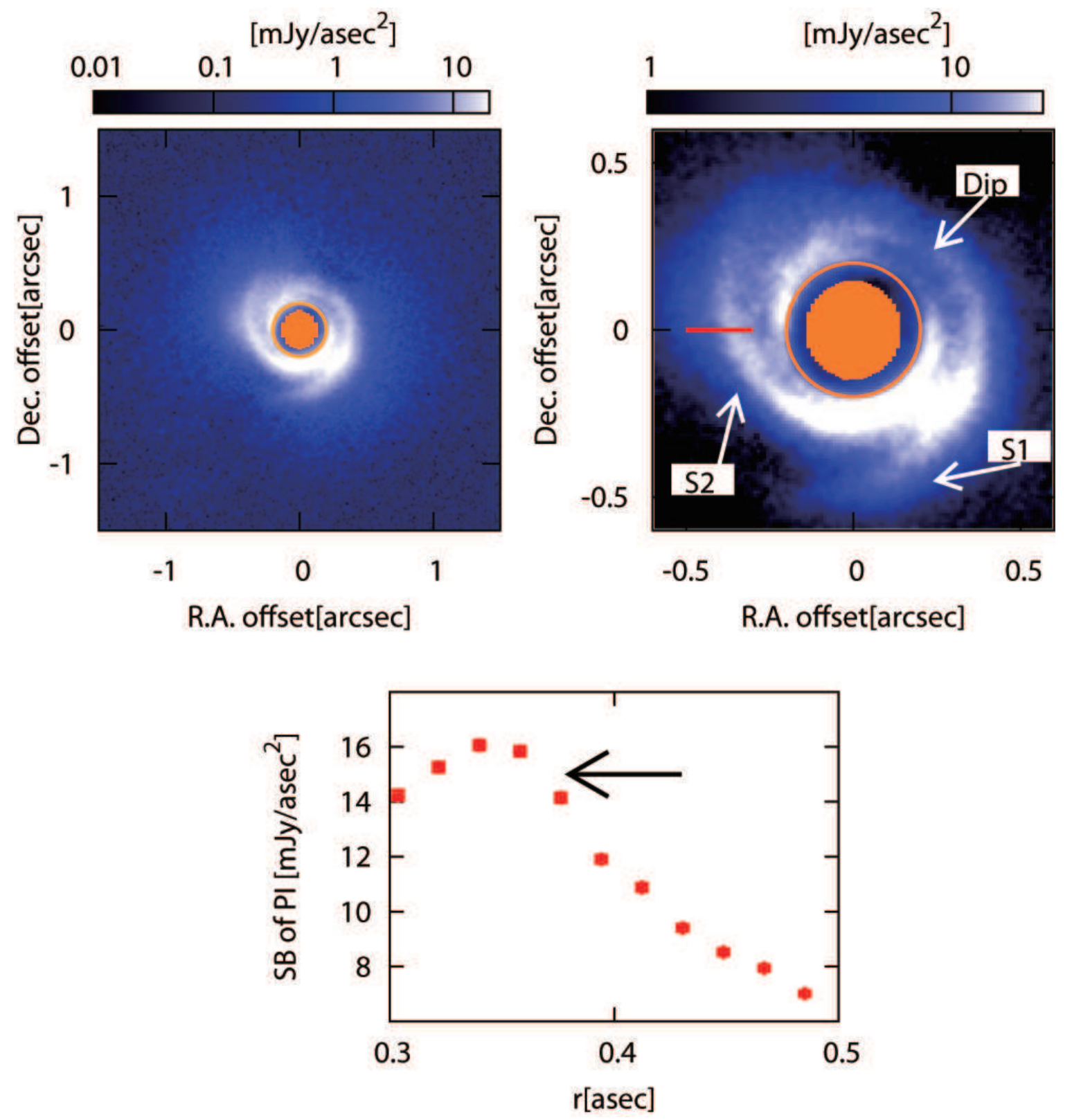

Fig. 2.- Top: PI image of SAO 206462 in the north-up configuration with log-stretch color scales. The filled orange circles at the center indicate the mask size $\left(r=00^{\prime \prime} 15\right)$. The circles around have $r=0 . \prime 2$, exterior to which the features are considered to be real. The right panel is central region's closeup. Different color scales are used to enhance the spirals labeled as "S1" and "S2". The "Dip" may be due to depolarization. Bottom: PI profile along the red line in the top right panel. The arrow indicates the location of S2. The position errors are not shown for visibility.

\subsection{Azimuthal and Radial Profiles}

Here, we summarize the overall disk structure exterior to the spirals. Figure 3 (top panels) shows the azimuthal SB profiles at $r=0^{\prime \prime} .5$ and $r=0^{\prime \prime} .7$. At $r \gtrsim 0^{\prime \prime} .5$, SB has maxima around position angle (PA) $\sim 50^{\circ}-60^{\circ}$ and $230^{\circ}-240^{\circ}$. Since the polarization is maximized at $\sim 90^{\circ}$ scattering (e.g., Graham et al. 2007), it is implied that the disk major axis is at $\mathrm{PA} \sim 50^{\circ}-60^{\circ}$, comparable to estimates by $\mathrm{CO}$ observations: $\mathrm{PA}=56^{\circ} \pm 2^{\circ}$ by Pontoppidan et al. (2008) and $64^{\circ} \pm 2^{\circ}$ by Lyo et al. (2011). We adopt PA $=55^{\circ}$ for the major axis and $i=11^{\circ}$ for inclination (see Section 1 ). Our spiral model fitting results (the next section) are little affected even if we assume a face-on geometry.

From CO observations, it is known that the south-west side is receding (e.g., Lyo et al. 2011). Therefore, either the north-west or south-east side is the near side. We do not see an obvious forward scattering excess in the NIR image. However, since the spirals are typically trailing, it is inferred that the south-east is the near side.

Figure 3 also shows the radial $P I$ profiles along the major axis, which is roughly consistent with $r^{-3}$ in the outer part, indicating a flat (not highly flared) disk (Whitney and Hartmann 1992). The radial slopes vary as PA from $\sim-2$ to $\sim-4.5$ (fitting at $0^{\prime \prime} .6<r<1^{\prime \prime} .0$ ) or from $\sim-2.5$ to $\sim-5$ (fitting at $0^{\prime \prime} .3<r<0$. 9 ), with shallower slopes typically appearing in the minor axis directions. However, $r^{-3}$ is representative on average. This slope is observed in several 

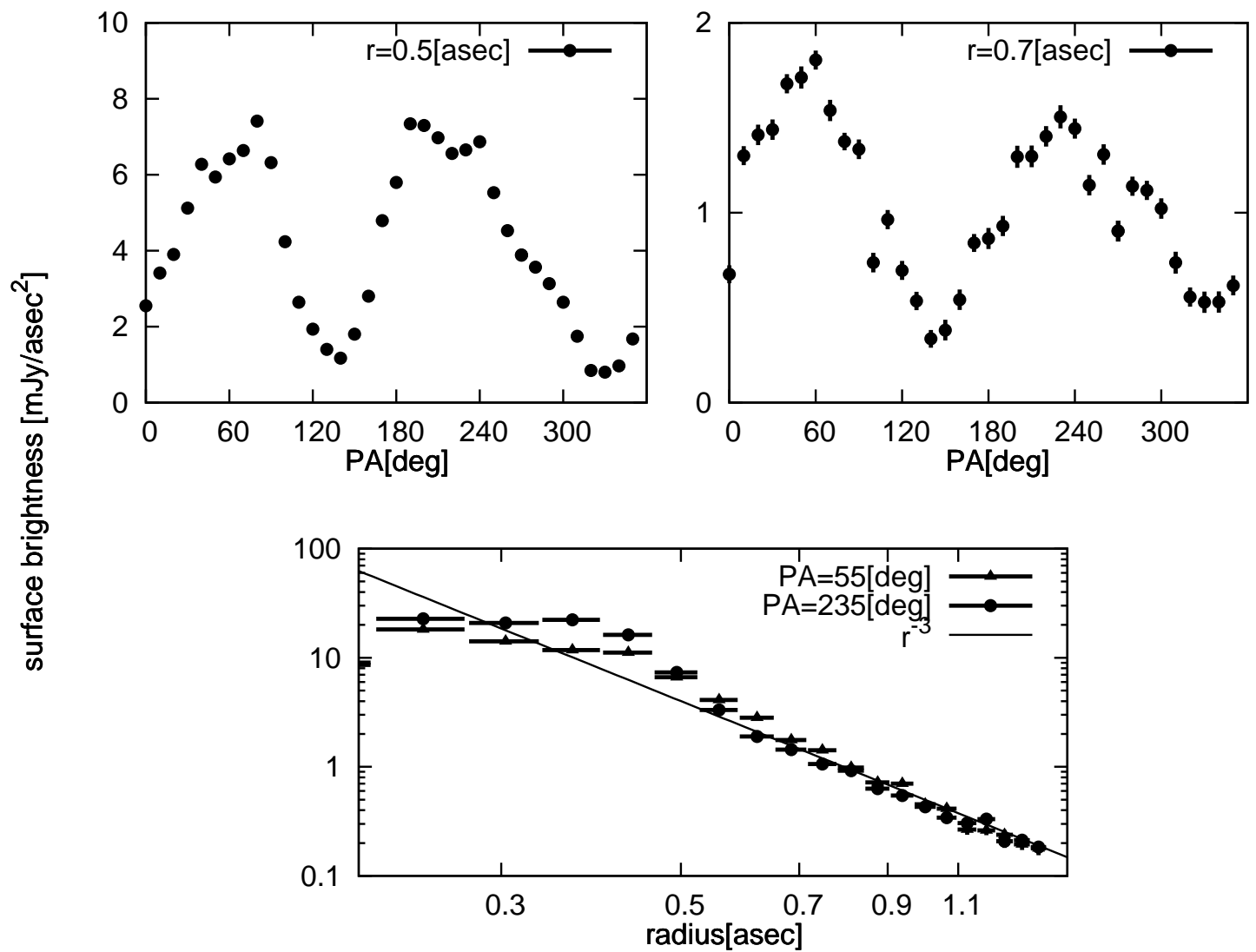

Fig. 3.- Top: Azimuthal SB profile at $r=0 . \prime 5$ (left) and $r=0^{\prime \prime} .7$ (right) with PA measured from north to east. Bottom: Radial profile along the major axis. Position errors indicate the FWHM of the PSF. SB errors are estimated using frame-by-frame deviations.

other HAeBe disks (e.g., Fukagawa et al. 2010, for total intensity data), although HD 97048 disk exhibits a shallower slope (Quanz et al. 2012).

\section{SPIRAL STRUCTURE MODELING}

Among several features in the image, the most interesting one is the non-axisymmetric spirals. In order to understand them, we propose a model based on the spiral density wave theory (e.g., Lin and Shu 1964; Goldreich and Tremaine 1978, 1979; Ogilvie and Lubow 2002), assuming that NIR emission traces the disk surface density structure. With such a model, the spiral structures can be used to infer the disk temperature, independently of, for example, CO line observations.

The shape of the spiral density wave is determined by the location of the launching point (corotation radius $r_{\mathrm{c}}$ ) and disk's thermal and rotation profiles. When the disk rotation angular frequency is $\Omega(r) \propto r^{-\alpha}$ and the sound speed is $c(r) \propto r^{-\beta}$, the shape of the wave far from $r_{\mathrm{c}}$ is given by

$$
\begin{aligned}
\theta(r)= & \theta_{0}-\frac{\operatorname{sgn}\left(r-r_{\mathrm{c}}\right)}{h_{\mathrm{c}}} \times \\
& {\left[\left(\frac{r}{r_{\mathrm{c}}}\right)^{1+\beta}\left\{\frac{1}{1+\beta}-\frac{1}{1-\alpha+\beta}\left(\frac{r}{r_{\mathrm{c}}}\right)^{-\alpha}\right\}-\left(\frac{1}{1+\beta}-\frac{1}{1-\alpha+\beta}\right)\right] }
\end{aligned}
$$

in the polar coordinate $(r, \theta)$, where $h_{\mathrm{c}}=c\left(r_{\mathrm{c}}\right) / r_{\mathrm{c}} \Omega\left(r_{\mathrm{c}}\right)$ denotes the disk aspect ratio at $r=r_{\mathrm{c}}$ and $\theta_{0}$ gives the phase. Equation (1) well approximates the shape of the density wave given by the WKB theory (Rafikov 2002; Muto et al. 2011). When the spiral is excited by a planet in a circular orbit, its location is $\sim\left(r_{\mathrm{c}}, \theta_{0}\right)$. Equation (10) has five parameters, $\left(r_{\mathrm{c}}, \theta_{0}, h_{\mathrm{c}}, \alpha, \beta\right)$.

Two non-axisymmetric features, S1 and S2, (Figure 4) are identified as follows. First, local maxima in the radial SB profiles normalized by $r^{2}$ (to take into account the dilution of the stellar flux) are traced at every $1^{\circ}$ step with data at $170^{\circ}<\mathrm{PA}<360^{\circ}(\mathrm{S} 1)$ and at $50^{\circ}<\mathrm{PA}<190^{\circ}(\mathrm{S} 2)$. The points near the minor axis $\left(\mathrm{PA} \sim 325^{\circ}\right)$ are excluded because the structure there may be affected by depolarization. The points at PA $>200^{\circ}(\mathrm{S} 1)$ and $\mathrm{PA}>114^{\circ}(\mathrm{S} 2)$ may be a part of axisymmetric rings since they are found to have constant radii. After removing these points, we have 27 (S1) and 56 (S2) points as representing samples of non-axisymmetric spirals, with the opening angle of $\sim 15^{\circ}$ for both S1 and S2. We estimate that the uncertainty of the location of the maxima is given by the FWHM of the PSF. 


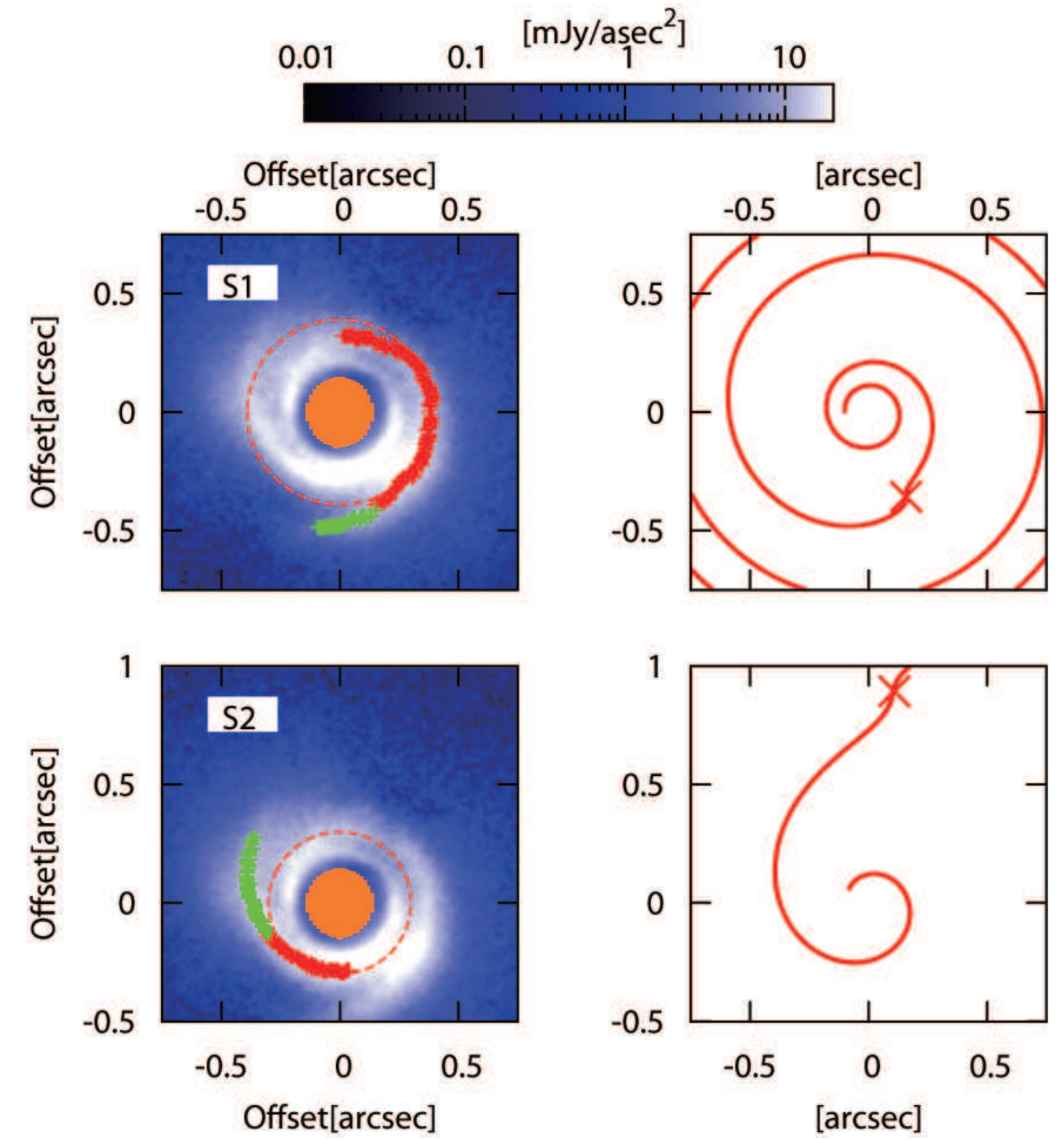

FIG. 4. - Left: Red and green points indicate the locations of the maxima of radial profiles for S1 (top) and S2 (bottom). The green ones are used for the fitting by Equation (1) (Section 4). Dashed lines indicate circles with $r=0^{\prime \prime} 39$ (top) and $r=00^{\prime \prime} 3$ (bottom). Right: The spiral shape given by Equation (1) with the best-fit parameters of $\mathrm{S} 1$ (top) and $\mathrm{S} 2$ (bottom). Crosses show the locations of $\left(r_{\mathrm{c}}, \theta_{0}\right)$.

In order to fit the non-axisymmetric structures by Equation (11), we fix $\alpha$ and $\beta$ at 1.5 (Kepler rotation) and 0.4, respectively, as in Lyo et al. (2011), while other parameters are varied as $\left(0{ }^{\prime \prime} 1<r_{\mathrm{c}}<00^{\prime \prime} 9,0<\theta_{0}<2 \pi, 0.05<h_{\mathrm{c}}<\right.$ $0.25)$. Note that different values of $\beta$ yield similar results. Since it is difficult to fit S1 and S2 simultaneously, they are fitted independently.

The "best-fit" parameters are $\left(r_{\mathrm{c}}, \theta_{0}, h_{\mathrm{c}}\right)=\left(0^{\prime \prime} 39,204^{\circ}, 0.08\right)$ for $\mathrm{S} 1$ (reduced $\left.\chi^{2}=0.52\right)$ and $\left(r_{\mathrm{c}}, \theta_{0}, h_{\mathrm{c}}\right)=$ $\left(0^{\prime \prime} 9,353^{\circ}, 0.24\right)$ for $\mathrm{S} 2$ (reduced $\left.\chi^{2}=0.31\right)$. The spiral shapes with these parameters are shown in Figure 4. However, the parameter degeneracy is significant. Figure 5 shows the parameter space of $\left(r_{c}, \theta_{0}\right)$ with $63.8 \%$ confidence level for $h_{\mathrm{c}}=0.1$ and $h_{\mathrm{c}}=0.2$. Note that in Figure 5, the "best-fit" of $\left(r_{\mathrm{c}}, \theta_{0}\right)$ is outside the domain of confidence in some cases because $h_{\mathrm{c}}$ is not the same as the best-fit. Despite the parameter degeneracy, the values of the aspect ratio which fit the shape of the spiral $\left(h_{\mathrm{c}} \sim 0.1\right)$ are consistent with those obtained from the sub-mm map of the disk (e.g., $h=0.096(r / 100 \mathrm{AU})^{0.15}$; Andrews et al. 2011).

The spiral density wave theory predicts that the pattern speed deviates from the local Kepler speed;

$$
\Omega_{\text {pattern }}=0.8\left(\frac{r_{\mathrm{c}}}{70 \mathrm{AU}}\right)^{-3 / 2}\left(\frac{M_{*}}{1.7 M_{\odot}}\right)^{1 / 2}[\mathrm{deg} / \mathrm{yr}]
$$




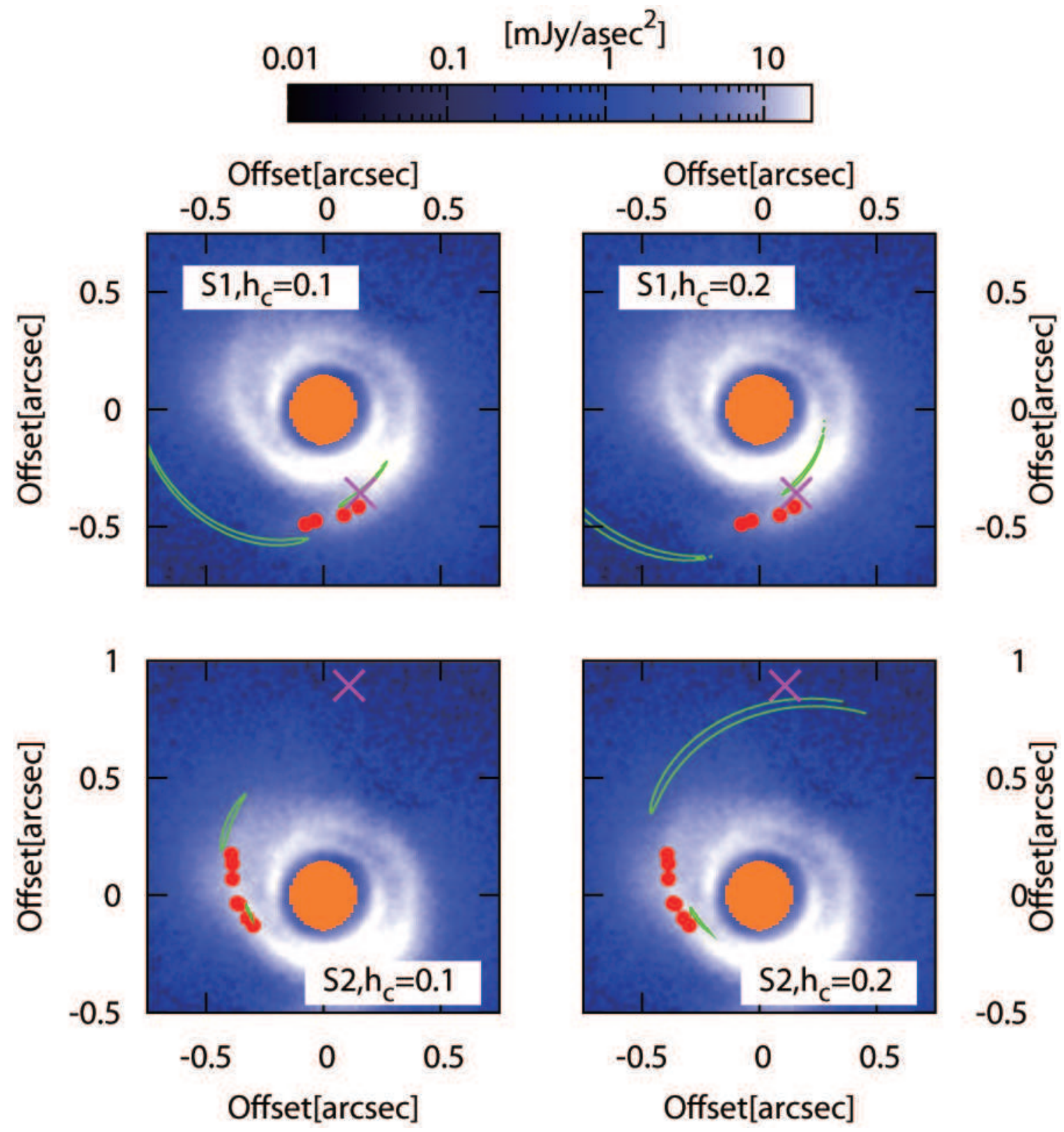

FIG. 5.- Parameter degeneracy of the fitting for S1 (top) and S2 (bottom) with $h_{\mathrm{c}}=0.1$ (left) and $h_{\mathrm{c}}=0.2$ (right). The red points show a part of the data fitted. The green curves indicate the $63.8 \%$ confidence level for the locations of $\left(r_{\mathrm{c}}, \theta_{0}\right)$. The magenta crosses are $\left(r_{\mathrm{c}}, \theta_{0}\right)$ for the best-fit parameters. Note that the best-fit values of $h_{\mathrm{c}}$ are different from those shown in the figure.

is not necessarily equal to $\Omega(r)$. When $r_{\mathrm{c}}=0^{\prime \prime} 5(\sim 70 \mathrm{AU})$, the spiral will move $\sim 10^{\circ}$ in a decade, corresponding to movement of $00^{\prime \prime} 1$. Considering the PSF scale of our observations and the locations of the spirals, such deviations can be detectable over a couple of decades. Moreover, if the two spirals have distinct corotation radii, their relative locations change in time due to the pattern speed difference. Such measurements will confirm that the observed feature is really the density wave, providing indisputable evidence of dynamical activity.

Note that it would be difficult to detect spirals in colder disks ( smaller $h_{\mathrm{c}}$ ), where spirals are more tightly wound, due to the blurring by the PSF. The lower detectable limit of $h_{\mathrm{c}}$ is typically $h_{\mathrm{c}} \sim 0.01-0.03$ for our set of parameters. The combination of high angular resolution and warm temperatures allows the spiral structure in the SAO 206462 disk to be resolved. Further spirals might be detectable in similarly warm disks.

\section{SUMMARY AND DISCUSSION}

In this Letter, we present a high-resolution image of the SAO 206462 transitional disk using Subaru/HiCIAO, with an inner working angle of $0^{\prime \prime} 2$. We discover non-axisymmetric spiral features, which can be explained by the spiral density wave theory with a reasonable value of the disk aspect ratio $\left(h_{\mathrm{c}} \sim 0.1\right)$. The model is robust in a sense that it does not assume the origin of such structure explicitly.

The detection of scattered light within the sub-mm cavity itself is interesting, since Andrews et al. (2011) predicts 
that the sub-mm cavity is heavily depleted. Our data in tandem with the millimeter data may suggest that the depletion of grains at different sizes is not uniform. Dong et al. (2012) discuss such discrepancies between sub-mm dust continuum images and NIR scattered light images in terms of differing spatial distributions as a function of grain size from a general theoretical perspective.

Our major assumption is that $P I$ at $H$-band, tracing the scattered light at the disk surface, actually traces the surface density variations. This assumption is valid when the disk is in vertical, isothermal hydrostatic equilibrium without rapid radial surface density variations (e.g., Muto 2011). Structures near the midplane are, however, preferentially observed at longer wavelengths with high spatial resolution: Atacama Large Millimeter/Submillimeter Array (ALMA) can be an ideal instrument.

Among several possible causes for the spiral structures (see also Hashimoto et al. 2011), one interesting idea is that planets excite them. In this case, the domain of possible locations of the planets is given by the green curves in Figure 5. If the two spirals have distinct corotation radii, there may be two (unseen) planets embedded in the disk. The amplitude of the surface density perturbation scales with the planet mass as $\delta \Sigma / \Sigma \sim G M_{\mathrm{p}} \Omega / c^{3}$ for non-gap-opening low-mass planets $\left(G M_{\mathrm{p}} \Omega / c^{3} \lesssim 1\right.$, e.g., Tanaka et al. 2002). In our data, the amplitude of the spiral wave is typically $\sim 30 \%$ (Figure 2), implying $M_{\mathrm{p}} \sim 0.5 M_{\mathrm{J}}$.

The typical error of $P I$ in our image is $\sim 5 \%$. If this is typical of HiCIAO, it is capable of detecting the indirect signatures of planets down to $M_{\mathrm{p}} \sim 0.05 M_{\mathrm{J}}$. ADI is promising in finding a point source; however, small field rotation $\left(\sim 13^{\circ}\right)$ due to the southerly declination $\left(-39^{\circ}\right)$ of SAO 206462 makes obtaining the total intensity difficult in our data. Future $L$-band observations may reveal thermal emission from a planet, if it exists, or its surrounding (accreting) gas.

The authors thank Roman Rafikov for comments, the support staff members of the IRTF and REM telescopes for assistance in obtaining the SED data, and the IR\&D program at The Aerospace Corporation. REM data in this study were obtained under Chilean National TAC programs CN2011A-050 and CN2011B-31. This work is partially supported by KAKENHI 22000005 (MT), 23103002 (MH and MH), 23103004 (MM and MF), 23103005, 23244027, 18540238 (SI), and 22.2942 (TM), WPI Initiative, MEXT, Japan (ELT), NSF AST 1008440 (CAG), 1009203 (JC), and 1009314 (JPW), and NASA NNH06CC28C (MLS) and NNX09AC73G (CAG and MLS). Part of this research was carried out at JPL.

\section{REFERENCES}

Andrews, S.M., Wilner, D.J., Espaillat, C., Hughes, A.M., Dullemond, C.P., McClure, M.K., Qi, C., \& Brown, J.M., 2011, ApJ, 732, 42

Brown, J.M. et al. 2007, ApJ, 664, L107.

Brown, J.M., Blake, G.A., Qi, C.. Dullemond, C.P., Wilner, D., Williams, J., Andrews, S., et al. 2008, ApJ, 704, 496.

Covino, S. et al., 2004, in Proc. SPIE, 5492, Ground-based Instrumentation for Astronomy, ed. Moorwood, A. F. M., \& Iye, M., 1613

Cushing, M.C., Vacca, W.D., \& Rayner, J.T. 2004, PASP, 116, 362

Dent, W. R. F., Greaves, J. S., \& Coulson, I. M., MNRAS, 2005, 359,663

Dong, R. et al., 2012, ApJ, accepted, arXiv:1203.1612

Doucet, C., Pantin, E., Lagage, P.O., Dullemond, C.P. 2006, A\&A 460, 117

Fedele, D., van den Ancker, M.E, Acke, B., van der Plas, G., van Boekel, R., Wittkowski, M., Henning, Th., Bouwman, J., Meeus, G., Rafanelli, P. 2008, A\&A 491, 809.

Fukagawa, M. et al., 2010, PASJ, 62, 347

Goldreich, P. \& Tremaine, S., 1978, ApJ, 222, 850

Goldreich, P. \& Tremaine, S., 1979, ApJ, 857, 871

Grady, C.A et al. 2009, ApJ, 699, 1822

Graham, J. R., Kalas, P. G., \& Matthews, B. C., ApJ, 654, 595

Hashimoto, J. et al. 2011, ApJ, 729, L17.

Hayano, Y. et al. 2004, in Proc. SPIE5490, Advancements in Adaptive Optics, ed. Calia, D. B., Ellerbroek, B. L., \& Ragazzoni, R., 1088

Hinkley, S. et al. 2009, ApJ, 701, 804

Hodapp, K. W. et al., 2008, in SPIE Conference Series 7014, Ground-based and Airborne Instrumentation for Astronomy II, ed. I. S. McLean, \& M. M. Casali, 701419

Joos, F., Buenzli, E., Schmid, H. M., Thalmann, C. et al. 2008, Proc. SPIE, 7016, 48

Lin, C. C. \& Shu, F. H, 1964, ApJ, 140, 646

Lyo, A-Ran, Ohashi, N., Qi, C., Wilner, D.J., Su, Y.-N., 2011, AJ, 142, 151

Mariñas, N., Telesco, C.M., Fischer, R.S., \& Packham, C. 2011, ApJ737, 57.
Minowa, Y. et al., 2010, in SPIE Conference Series 7736, Adaptive Optics Systems II, ed. Ellerbroek, B., L., Hart, M., Hubin, N. \& Wizinowich, P. L., 77363N

Müller, A., van den Ancker, M.E., Launhardt, R., Pott, J.U., Fedele, D., \& Henning, Th. 2011, A\&A 530, 85.

Muto, T., 2011, ApJ, 739, 10

Muto, T., Suzuki, T. K., \& Inutsuka, S., 2010 ApJ, 724, 448

Ogilvie, G. I., \& Lubow, S. H., MNRAS, 2002, 330, 950

Perrin, M. D., Schneider, G., Duchene, G., Pinte, C., Grady, C. A., Wisniewski, J. P., \& Hines, D., ApJ, 2009, 707, L132

Pontoppidan, K.M., Blake, G.A., van Dishoeck, E.F., Smette, A., Ireland, M.J., \& Brown, J. 2008, ApJ, 684, 1323.

Quanz, S. P., Schmid, H.M., Geissler, K., Meyer, M.R., Henning, Th., Brandner, W., \& Wolf, S. 2011a, ApJ, 738, 23

Quanz, S. P., Birkmann, S. M., Apai, D., Wolf, S., \& Henning, T., 2012, A\&A, 538, id.A92

Rafikov, R. R., 2002, ApJ, 569, 997

Rayner, J. T., Cushing, M.C., \& Vacca, W.D. 2009, ApJS, 185, 289

Sitko, M.L., et al, 2012, ApJ, 745, id.29

Suzuki, R. et al., 2010, in SPIE Conference Series 7735, Ground-based and Airborne Instrumentation for Astronomy III, ed. I. S. McLean, Ramsay, S. K., \& H. Takami, 773530

Tamura, M. et al. 2006, Proc. SPIE, 6269, 28

Tamura, M. 2009, in proc. "Exoplanets and Disks: Their Formation and Diversity" (2009 March 9-12), eds. Usuda, T., Ishii, M. and Tamura, M. (National Astronomical Observatory of Japan), p. 11-16

Tanaka, H., Takeuchi, T., \& Ward, W. R., 2002, ApJ, 565, 1257

Tokunaga, A. T., Simons, D. A., \& Vacca, W. D., 2002, PASP, 114,180

Thalmann, C. et al. 2010, ApJ, 718, L87.

Vacca, W.D., Cushing, M.C. \& Rayner, J. T. 2003, PASP, 115, 389

van der Plas, G., van der Ancker, M., E., Fedele, D., Acke, B., Dominik, C., Waters, L. B. F. M., \& Bouwman, J., 2008, A\&A, 485,487

Whitney, B. A., \& Hartmann, L., 1992, ApJ, 395, 529 